\title{
Search for novel STAT3-dependent genes reveals SERPINA3 as a new STAT3 target that regulates invasion of human melanoma cells
}

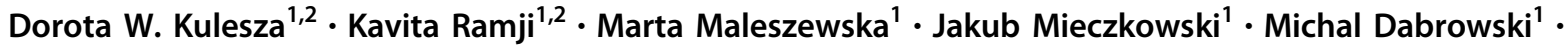 \\ Salem Chouaib ${ }^{3} \cdot$ Bozena Kaminska $\oplus^{1}$
}

Received: 4 January 2019 / Revised: 19 April 2019 / Accepted: 5 June 2019 / Published online: 5 July 2019

(c) The Author(s), under exclusive licence to United States and Canadian Academy of Pathology 2019

\begin{abstract}
Transcription factor signal transducer and activator of transcription 3 (STAT3) is constitutively activated in many cancers and promotes uncontrolled tumor growth and progression through multiple mechanisms. Compelling evidence shows tissue and cell-specific sets of STAT3 targets. Transcriptional targets of STAT3 in melanoma cells are largely unknown. Malignant melanoma is a deadly disease with highly aggressive and drug-resistant behavior. Less than $10 \%$ of patients with advanced melanomas reach the 5-year survival, partly due to the aggressive character of the tumor and ineffectiveness of current therapeutics for treating metastatic melanoma. STAT3 is constitutively activated in melanoma cells and plays important roles in its growth and angiogenesis in tumor xenograft studies. Moreover, highly metastatic melanoma cells have higher levels of active STAT3 than poorly metastatic ones. To identify genes that are driven by STAT3 in human melanoma cells, we performed JAK/STAT signaling specific and global gene expression profiling of human melanoma cells with silenced STAT3 expression. For selected genes, we performed computational identification of putative STAT3-binding sites and validated direct interactions STAT3 with defined promoters by using chromatin immunoprecipitation followed by qPCR. We found that STAT3 knockdown does not affect human melanoma cell viability, proliferation, or response to chemotherapeutics. We show that STAT3 regulates a discrete set of genes in melanoma cells, including SERPINA3, a novel STAT3 target gene, which is functionally involved in regulation of melanoma migration and invasion. Knockdown of STAT3 impaired cell migration and invasion, in part via regulation of its transcriptional target SERPINA3. Our results present novel targets and functions of STAT3 in melanoma cells.
\end{abstract}

\section{Introduction}

Signal transducer and activator of transcription 3 (STAT3) belongs to a family of STAT transcription factors that

These authors contributed equally: Dorota W. Kulesza, Kavita Ramji

Supplementary information The online version of this article (https:// doi.org/10.1038/s41374-019-0288-8) contains supplementary material, which is available to authorized users.

Bozena Kaminska

b.kaminska@nencki.gov.pl

1 Laboratory of Molecular Neurobiology, Neurobiology Center, The Nencki Institute of Experimental Biology, Warsaw, Poland

2 Postgraduate School of Molecular Medicine, Warsaw Medical University, Warsaw, Poland

3 INSERM UMR1186, Integrative Tumor Immunology and Genetic Oncology, Institute Gustave Roussy, Viallejif, France mediate cytokine signaling, and are positively or negatively involved in the initiation, propagation, and resolution of immune responses [1,2]. Phosphorylation of STAT3 at tyrosine 705 leads to its activation, dimerization, nuclear translocation, and binding to DNA, which regulates gene transcription. STAT3 can directly and indirectly regulate the expression of many genes implicated in survival (survivin, bcl-xl, mcl-1, FLICE-like inhibitory protein), cell-cycle progression (c-fos, c-myc, cyclin D1), invasion (matrix metalloproteinase $M M P 2,9,14$ ), and angiogenesis (vascular endothelial growth factor, VEGF) [3-9].

Aberrant signaling of STAT3 has been implicated in malignant transformation and tumor progression [10, 11]. Constitutive activation of STAT3 occurs in various human cancers, including melanomas [11]. Preclinical studies using antisense RNA, RNA interference, dominant negative STAT3, and small-molecule inhibitors indicated that blockade of STAT3 signaling leads to suppression of tumor cell growth or induction of apoptosis in vitro, and in animal 
models of prostate, head and neck, pancreatic, and lung cancer [12-18]. However, other studies showed no impact of STAT3 signaling on survival of cancer cells $[19,20]$ or the suppressive role of STAT3, depending on genomic alterations in glioma cells [21, 22].

Malignant melanoma is a deadly disease with highly aggressive and drug-resistant behavior. Less than $10 \%$ of patients with advanced melanomas reach the 5-year survival, partly due to the aggressive character of the tumor and ineffectiveness of current therapeutics for treating metastatic melanoma [23]. STAT3 is constitutively activated in melanoma cells and contributes to melanoma growth and tumor angiogenesis in xenografts $[11,12,24]$. Moreover, highly metastatic melanoma cells have higher levels of p-STAT3 than poorly metastatic ones or benign melanocytic nevi [25]. Blocking activated p-STAT3 in metastatic melanoma cells, suppressed the invasiveness and tumor growth in nude mice implicating p-STAT3 in tumor progression [26]. p-STAT3 levels are higher in brain metastases $(81 \%$ express activated STAT3) than in cutaneous primary melanomas [27]. While the abundance of p-STAT3 was not predictive of development or time to brain metastases, p-STAT3 expression was found to be a negative prognostic factor for overall survival in melanoma patients that did not develop such metastases [27, 28]. STAT3 activation contributes to dysregulated expression of factors implicated in tumorigenesis: basic fibroblast growth factor (bFGF), vascular endothelial growth factor (VEGF), and matrix metalloproteinase-2 (MMP-2), affecting angiogenesis and invasion of melanoma cells [26]. Better understanding of STAT3 involvement in molecular mechanisms of melanoma formation and progression may result in more efficient therapies. Transcriptional targets of STAT3 are largely unknown in melanoma cells. A meta-analysis of 29 ChIP-seq data sets, covering genomewide occupancy of various STATs in different cell types (macrophages, $\mathrm{B}$ and $\mathrm{T}$ lymphocytes, murine embryonic fibroblasts, embryonic and liver cells) indicate that the majority of STAT-binding sites are cell-type specific [29]. Pharmacological inhibition of STAT3 with a bioactive flavonoid apigenin inhibited cell migration and invasion in human and murine melanoma cells along with downregulation of some STAT3 target genes MMP-2, MMP-9, $V E G F$, and Twistl [30].

In this study, we aimed at resolving the role of STAT3 in melanoma cells and identification of STAT3 direct targets. We studied if knockdown of STAT3 expression affects functional properties of human melanoma cells, and performed a pathway specific and global gene expression profiling to identify novel direct STAT3 targets in melanoma cells.

\section{Materials and methods}

\section{Cell lines, culture conditions, and treatments}

Human melanoma cell lines: WM 902B (primary melanoma in the vertical growth phase) and WM 239A (metastatic melanoma) were derived from melanoma patients in the Greater Poland Oncology Center, Poland; G1, ME260, T291A, ME290 cells were derived from metastatic melanoma patients in the Institute Gustave Roussy, France. Cells were cultured in the RPMI 1640 medium supplemented with 10\% FBS (Sigma, Chemical Co., St. Louis, MO) and antibiotics (50 U/ml penicillin and $50 \mathrm{~g} / \mathrm{ml}$ streptomycin), in a humidified atmosphere of $\mathrm{CO}_{2}$ /air $(5 \% / 95 \%)$ at $37^{\circ} \mathrm{C}$. Melanoma cells were passaged weekly, and seeded for experiments at density $10^{5}$ cells/plate as determined using NucleoCounter (Chemotec). Normal human epidermal melanocytes (NHEM, Lonza) were cultured in $\mathrm{MBM}^{\mathrm{TM}}-4$ melanocyte basal medium (CC-3249, Lonza) supplemented with Endothelin-3 (CC-4510, Lonza) in a humidified atmosphere of $\mathrm{CO}_{2} / \mathrm{air}(5 \% / 95 \%)$ at $37^{\circ} \mathrm{C}$. Cells were treated with specific compounds or medium $48 \mathrm{~h}$ after transfection. In some experiments, $5 \mu \mathrm{M}$ concentration doxorubicin (Sigma-Aldrich, Munich, Germany) or UVC irradiation with 15 -s pulse of $100 \mathrm{~W}$ UVC were used.

\section{Transfection of cells}

Melanoma cells were transfected by Amaxa electroporation with control siRNA or STAT3 siRNA (Santa Cruz Biotechnology, Santa Cruz, CA, USA) at the final concentration of $150 \mathrm{nM}$ using the Cell Line Nucleofector Kit V (Lonza). STAT3 siRNA was a mixture of three different siRNAs specific for STAT3. The total proteins and RNA were isolated 48 or $72 \mathrm{~h}$ after the transfection.

\section{Western blot analysis}

Whole-cell protein extracts were prepared as previously described [20]. Cells were collected in a lysis buffer with protease inhibitors $(20 \mathrm{mM}$ Tris- $\mathrm{HCl}, 137 \mathrm{mM} \mathrm{NaCl}, 25$ $\mathrm{mM}$ beta-glycerophosphate, $2 \mathrm{mM}$ NaPPi, $2 \mathrm{mM}$ EDTA, 1 $\mathrm{mM} \mathrm{Na} \mathrm{VO}_{4}, 1 \%$ Triton-X, $10 \%$ glycerol, $0.5 \mathrm{mM}$ DTT, 1 $\mathrm{mM}$ PMSF, $15 \mu \mathrm{g} / \mathrm{ml}$ aprotinin and leupeptin, $2 \mathrm{mM}$ benzamidine). Protein samples were resolved by SDS-PAGE, and transferred to hybond ECL nitrocellulose membranes (GE Healthcare Amersham, Buckinghamshire, UK). Antibodies recognizing phospho-STAT3 (Tyr705), total STAT3, and cleaved PARP were obtained from Cell Signaling (Beverly, MA, USA). Anti-cyclinD1 antibody was obtained from Santa Cruz Biotechnology (Santa Cruz, CA, USA) and anti- $\beta$-actin-peroxidase-conjugated antibody was 
from Sigma-Aldrich (Munich, Germany). Anti-SERPINA3 antibody (LS-B5826) was from LIFESPAN Biosciences. Membranes were incubated with primary antibodies diluted in TBS-T $(10 \mathrm{mM}$ Tris/ $\mathrm{HCl}, \mathrm{pH} 8.0,0.12 \mathrm{M} \mathrm{NaCl}, 0.1 \%$ Tween-20, and $0.05 \%$ sodium azide) containing 5\% skimmed milk. Antibody recognition was detected with the respective secondary antibody either anti-mouse IgG, antirabbit IgG linked to horseradish peroxidase (Cell Signaling Technology, Beverly, MA, USA). Immunocomplexes were visualized using the enhanced chemiluminescence detection system (Thermo Scientific, Rockford, USA).

\section{Analyses of cell viability, cell cycle, and apoptosis}

Cell survival was evaluated with a MTT metabolism test. Cells were incubated for $4 \mathrm{~h}$ with MTT (Sigma-Aldrich (Munich, Germany) followed by application of the lysis buffer containing 20\% SDS and 50\% DMF. Optical densities were measured at $570 \mathrm{~nm}$ using a scanning multiwell spectrophotometer.

Cell proliferation was determined with a Cell Proliferation ELISA BrdU assay (Roche Diagnostics GmbH, Mannheim, Germany). Cells were cultured in a 96-wells plate $\left(12 \times 10^{3}\right.$ cells/well $)$ and $24 \mathrm{~h}$ after specific treatments, BrdU labeling solution was added to each well for $2 \mathrm{~h}$. Cells were fixed, incubated with a mouse monoclonal anti-BrdU antibody conjugated with peroxidase, and after addition of $1 \mathrm{M} \mathrm{H}_{2} \mathrm{SO}_{4}$ reaction products were determined at $450 \mathrm{~nm}$ with Thermo labsystem Multiscan EX.

For cell cycle analysis, cells were fixed with ice-cold $70 \%$ ethanol $24 \mathrm{~h}$ after specific treatments, and stained with propidium iodide. Measurements of the DNA content (for evaluation of the cell cycle profile and percentages of apoptotic cells) were performed using a FACScalibur flow cytometer (Becton Dickinson). Percentages of cells in the sub-G1, G1, S, and G2/M phases were determined with CellQuest software (Becton Dickinson).

\section{RNA isolation and real-time PCR (qPCR) analysis}

The total RNA was isolated using The High Pure RNA Isolation Kit (Qiagen). Quality and quantity of the total RNA were verified by the Agilent 2100 Bioanalyzer electrophoresis. The total RNA was used to synthesize complementary DNA by extension of oligo d(T) 15 primers with M-MLV reverse transcriptase (Sigma-Aldrich). The TaqMan Array Human Cyclins and Cell Cycle Regulation (contains 44 different genes and 4 endogenous controls) and The TaqMan Array Human JAK-STAT Pathway (contains 92 different genes and 4 endogenous) from Applied Biosystems (Germany) were employed. PCR amplifications were performed in triplicate in a $10 \mu \mathrm{l}$ of reaction volume. For validation of the results of microarrays and TaqMan
Array profilers, FAM-labeled primers from Applied Biosystems and 2x TaqMan PCR Master Mix were used. For evaluation of STAT3 silencing primers: 5'-ATTGCCCG GATTGTGGCCCG-3', 5'-CTCCGTCACCACGGCTGCT G-3', and 2x SybrGreen PCR MasterMix were used. The amount of target mRNA was first normalized to the $18 \mathrm{~S}$ RNA expression level in the same sample and then to an untreated control. Real-time PCR amplifications were performed in duplicate on complementary DNA equivalent to $25 \mathrm{ng}$ of RNA in $20 \mu \mathrm{l}$ of reaction mixture containing $2 \mathrm{X}$ SybrGreen PCR Master Mix (Applied Biosystems, Darmstadt, Germany).

\section{Global gene expression profiling and data analysis}

The total RNA was isolated and analyzed as described above. RNA samples were hybridized to Illumina Human HT12 v4 Beadarrays at the Cambridge Genomic Center, UK. The raw data were imported into $\mathrm{R}$ and analyzed with the Bioconductor beadarray package [31]. The data were further visually inspected for quality check, and normalized by quantile normalization. Annotation of probe sets was performed with information provided in the Ensembl database. Expression data analysis was performed using the $\mathrm{R}$ software environment for statistical computing. Using illuminaHumanv4.db Bioconductor package, we removed all probes matching repeat sequences, intergenic or intronic regions, and probes that does not match any genomic region or transcript. Differential expression analysis was performed using limma. A particular gene was recognized as significantly differentially expressed if its FDR was less than 0.05 and absolute value of its log (fold change) was higher than 1.4. The GOstats package was used to assign genes to Gene Ontology terms.

\section{Computational prediction of STAT-binding sites}

Promoter regions $(+/-10-\mathrm{kb}$ flanks of transcription start sites) of differentially expressed genes were searched for putative STAT3-binding sites (instances of STAT3-binding motifs) using the MatInspector and Genomatix Motif Family Library v.8.2, with the default optimized thresholds. Due to the well-known similarity of binding sites for all STAT proteins, instances of any motif from the V\$STAT family of motifs (thereafter referred to as STAT-binding motifs) were treated the same, i.e., as a potential STAT3binding site. To reduce false-positive motifs, for further analysis, we selected STAT motifs that overlap regions with experimental marks of active cis-regulatory regions in the Ensembl funcgen database, e.g., transcription factor-binding sites, DNase1 hypersensitivity, or activating epigenetic modifications (H3K27ac, H3K9me1). The above data were obtained in different cell types than used in this work, but 
some of them may be functional also in melanoma cells under study. The intersections between the STAT motifs and the funcgen data were obtained from the Nencki Genomics Database [32].

\section{Chromatin immunoprecipitation (ChIP) and qPCR}

ChIP experiments were performed on WM239 and G1 melanoma cells. Overall, $1 \times 10^{7}$ cells were fixed with $1 \%$ formaldehyde for $20 \mathrm{~min}$, and sonicated for number of pulses and time using Diagenode sonicator with components of ChIP IT Express kit (Active Motif, USA). DNA fragmentation was evaluated using Agilent Bioanalyzer. Each DNA sample (50 ng) was immunoprecipitated for $20 \mathrm{~h}$ at $4{ }^{\circ} \mathrm{C}$ with $1 \mu \mathrm{g}$ of the following ChIP quality antibodies: anti-P-STAT3, anti-STAT3 (Santa Cruz Biotechnology), or normal rabbit IgG (Calbiochem) which served as a negative control for IP reaction.

Immunoprecipitated DNA fragments were used as templates for PCR with the primers shown in Table 1:

The PCR reaction was started with an initial melt step at $94{ }^{\circ} \mathrm{C}$ for $4 \mathrm{~min}$ and then 30 cycles of $\left[94^{\circ} \mathrm{C}\right.$ for $20 \mathrm{~s}, 55^{\circ} \mathrm{C}$ for $30 \mathrm{~s}$, and $72{ }^{\circ} \mathrm{C}$ for $30 \mathrm{~s}$ ] and a hold cycle at $4{ }^{\circ} \mathrm{C}$ were performed. All PCR products were analyzed by resolving on $2 \%$ agarose gels with a 100 bp DNA ladder (Fermentas) as a standard.

\section{Scratch assay}

For a scratch assay, WM239 and G1 cells were plated in a six-well plate and cultured overnight in the DMEM containing $10 \%$ of FBS to reach $90 \%$ confluence. Cell monolayer was wounded with a sterile Eppendorf pipette tip $(100 \mu \mathrm{l})$, washed with PBS, then fresh medium was added. Pictures were taken immediately and after $18 \mathrm{~h}$.

Table 1 PCR primers used in this study

\begin{tabular}{|c|c|}
\hline Gene & Primer sequence \\
\hline \multirow[t]{2}{*}{$P S P H$} & $5^{\prime}-C T C A C A G C A C C G C A T G A T T C-3^{\prime}$ \\
\hline & 5'-CTCGCGGTTTGTTCCGTTTC-3' \\
\hline \multirow[t]{2}{*}{$G L D C$} & 5'-GCTGCCATCTCCAGTCCTAC-3' \\
\hline & 5'-GAACTTGGCCCGGAGTCTTT-3' \\
\hline \multirow[t]{2}{*}{$T P M T$} & $5^{\prime}-G A A T T C C T G G G A G C C C T A G-3^{\prime}$ \\
\hline & $5^{\prime}-C A G C T G T A A G C G A G G T A G G C-3^{\prime}$ \\
\hline \multirow[t]{2}{*}{$N N M T$} & $5^{\prime}-A G C G A A T T C T C T G G C T T G C T-3^{\prime}$ \\
\hline & $5^{\prime}-A A T G C T G A C C T G C A T A C C C C-3^{\prime}$ \\
\hline \multirow[t]{2}{*}{ LSM10 } & $5^{\prime}-C G C G T C A C G G A G A A A C T A C A-3^{\prime}$ \\
\hline & $5^{\prime}-A A G A G A C T C C A A T G A C G C A G G-3^{\prime}$ \\
\hline \multirow[t]{2}{*}{ SERPINA3 } & 5'-CTGACACCTTGCTTCCCAGT -3' \\
\hline & 5'-CACAAAGGCTGGCACTGTTC-3' \\
\hline
\end{tabular}

After washing with PBS, cells were fixed with $2 \%$ paraformaldehyde, stained with DAPI $(0.01 \mathrm{mg} / \mathrm{ml}$, Sigma), and visualized by fluorescent microscopy. The nuclei of cells migrating to the cell-free area of scratch were captured (10 photos were taken per condition) along the scratch. For analysis of cell migration, pictures were analyzed using ImageJ.

\section{Invasion assay}

Cancer cell invasion assays were performed using 24-well tissue culture inserts $(8-\mu \mathrm{m}$ pore size Transwell, Corning, NY, USA) coated with the Growth Factor Reduced Matrigel $^{\text {TM }}$ Matrix (BD Biosciences, San Diego, CA, USA). Matrigel $^{\mathrm{TM}}$ Matrix $(100 \mu \mathrm{l}$ of $1 \mathrm{mg} / \mathrm{ml}$ mixture) was diluted in distilled water, added to the inserts and dried under sterile conditions $\left(37^{\circ} \mathrm{C}\right)$ for $4-5 \mathrm{~h}$, and reconstituted for $30 \mathrm{~min}$ in $200 \mu$ of culture medium. WM239 and G1 cells (transfected with either control or siRNA against STAT3) were seeded as $5 \times 10^{4}$ cells/insert on Matrigel-covered membrane. After $18 \mathrm{~h}$, the Matrigel was scraped, and the membranes were washes with PBS and cells fixed with $95 \%$ methanol. Cell nuclei were stained with DAPI (4',6-diamidino-2-phenylindole; $0.01 \mathrm{mg} / \mathrm{ml}$, Sigma). The membranes from Transwell ${ }^{\circ}$ inserts were cut out and placed on a glass slide. Total numbers of invading cells that migrated through the Matrigel were determined using Laser Scanning Cytometry (LSC, CompuCyte).

\section{Statistical analysis}

All experiments have been performed at least three times on independent cell passages, in triplicates. Statistical analysis was done by Student's $t$ test, values $* p<0.05, * * * p<0.001$ are considered to be significant.

\section{Results}

\section{The effect of STAT3 silencing on expression of the JAK/STAT3 signaling pathway and the cell cycle regulation genes}

First, we analyzed levels of the total and phosphorylated STAT3 by western blotting in four human melanoma cell lines. The levels of phosphorylated STAT3 (phosphoSTAT3-Y705) were higher in metastatic melanoma cells (G1, WM239) in comparison with primary melanoma cells (T1, WM902) (Fig. 1a). To elucidate the role of STAT3 in melanoma cells, three melanoma cell lines exhibiting high levels of phospho-STAT3 were transfected with control or STAT3-specific siRNA. Knockdown of STAT3 was evaluated at the mRNA level by qPCR (Fig. 1b) and at the 

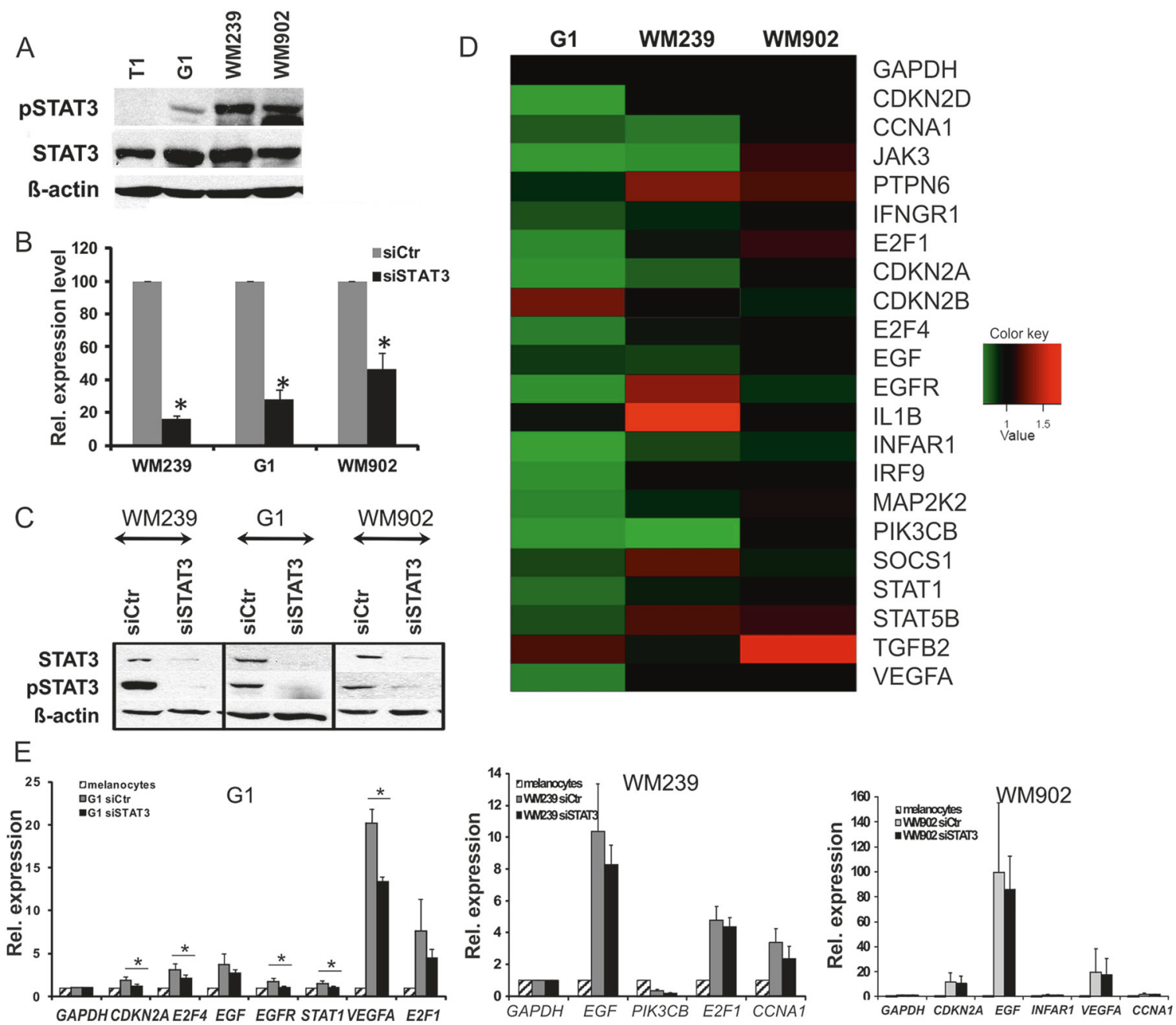

Fig. 1 The levels of phosphorylated STAT3 and the effects of STAT3 knockdown on the JAK/STAT signaling pathway/cell cycle-regulated gene expression in human melanoma cells. a Representative immunoblot shows levels of phosphorylated STAT3 (STAT3-Y705) and the total STAT3 in four melanoma cell lines: T1-primary melanoma; G1metastatic melanoma (from the same patient as T1 cells); WM902primary melanoma; WM239-metastatic melanoma. b, c Efficacy of STAT3 knockdown with siRNA was determined after $48 \mathrm{~h}$ at mRNA level by qPCR (b) and protein level by western blotting (c). Equal loading of total proteins was verified by re-probing of the membranes with antibody recognizing $\beta$-ACTIN. d Gene expression profiling with pathway-specific arrays. The total RNA was isolated from three melanoma cell lines transfected for $48 \mathrm{~h}$ with siRNA Ctr or siRNA
STAT3, and subjected to qPCR expression profiling with the human JAK/STAT signaling pathway and Cell cycle PCR profiler. Heatmap represents changes in expression levels after STAT3 knockdown related to respective control groups. Levels of gene expression are indicated on a color scale, with red representing upregulation and green downregulation of gene expression, black shows no changes. For a given gene, an average value from three experiments was computed. e Expression of selected genes was validated by qPCR at $48 \mathrm{~h}$ after the transfection, $n=3$. The bars represent average fold change of expression $+/-$ standard deviation (s.d.), fold change was related to the gene expression in melanocytes (three experiments, each in triplicate). Statistical analysis was done by Student's $t$ test, $p<0.05$ are considered to be significant

after STAT3 knockdown. All changes have been related to cells transfected with the control siRNA. Black color reflects no change in comparison with control siRNAtreated cells (i.e., the expression of housekeeping gene GAPDH did not change); green and red colors mark, respectively, downregulation or upregulation of gene expression in STAT3-depleted cells versus controls. The initial screening revealed a set of 21 most altered genes between STAT3-depleted (siSTAT3) and control (siCtr) melanoma cells. 
The most prominent changes in the expression of the investigated genes were observed in metastatic melanoma cell lines: G1 and WM239, while lesser alterations were noticed in WM902 primary melanoma cells (Fig. 1d). Only several genes demonstrated significantly different expression after validation with qPCR. In G1 melanoma cells, STAT3 knockdown led to the significant decrease in the expression of $C D K N 2 A, C D K N 2 D, E 2 F 4, E G F R$, IL1 $\beta$, INFAR1, IRF9, MAP2K2, PIK3CB, and VEGF mRNA levels. The levels of CCNA1, JAK3, IFNGR1, EGF, $P I K 3 C B$, and mRNA were significantly altered by STAT3 knockdown in WM239 melanoma cells, and the expression of TGF $\beta 2$ was significantly increased in primary melanoma WM902 cells upon STAT3 silencing. Comparison of the levels of selected genes to their expression in normal melanocytes revealed that the expression of all analyzed genes (except PIK3CB in WM239 cells) was significantly lower in melanocytes than in melanoma cells, and STAT3 knockdown led to partial restoration of their gene expression to the levels occurring in melanocytes (Fig. 1e).

\section{The effect of STAT3 knockdown on cell proliferation, survival, and susceptibility to cytotoxic treatments of melanoma cells}

The results presented above demonstrated that many genes implicated in the regulation of cell growth, cell cycle, and viability are downregulated by STAT3 knockdown. Therefore, we determined the effects of STAT3 knockdown on proliferation, viability, and response of melanoma cells to therapeutics. STAT3 knockdown did not affect cell viability of cultured human melanoma cells, as evidenced by MTT metabolism test (except for the slight reduction in G1 cells) and cell proliferation was moderately reduced in STAT3-depleted WM239 and WM902 cells in comparison with control cells, as demonstrated by BrdU proliferation assay (Fig. 2a). The analysis of percentages of cells with specific DNA content by flow cytometry showed that STAT3 knockdown has little impact on the cell-cycle distribution (Fig. 2b).

Furthermore, we examined the effects of STAT3 knockdown on sensitivity of melanoma cells to doxorubicin and UVC radiation. Melanoma cells depleted of STAT3 were similarly susceptible to doxorubicin-mediated death and UVC radiation as controls (Fig. 2c). A slight increase (about 20\%) in sensitivity of STAT3-depleted cells to UVC radiation was observed when BrDU incorporation was determined (Fig. 2c). Western blotting analysis of the levels of a cleaved PARP (an apoptosis marker) and Cyclin D1 (a cell cycle regulatory protein) did not reveal any difference in sensitivity of control and siSTAT3-transfected cells to the treatments (Fig. 2d). Altogether, the results show that STAT3 knockdown in two melanoma cell lines does not significantly affect basal cell viability, and only moderately modulates cell proliferation and sensitivity of melanoma cells to UVC.

\section{Global analysis of STAT3-dependent transcriptional network}

In order to investigate global changes in transcriptomes of melanoma cells, we performed microarray analysis of gene expression in control (siCtr) and STAT3-depleted (siSTAT3) WM239 and G1 melanoma cells. Heatmaps represent levels of differentially expressed genes in each cell line [ $p$-value $<0.05 ; \mathrm{FC}<1.4 \mathrm{v}$ FC $<0.7]$ (Fig. 3a). Statistically significant, STAT3-dependent changes were detected in the expression of 30 genes in WM239 cells and 11 in G1 cells. A set of common genes with altered expression in both cell lines was selected. Figure $3 \mathrm{~b}$ presents a barplot of genes more than $10 \%$ downregulated in both cell lines or $30 \%$ downregulated in at least one of treated cell lines compared with control cells (control siRNA).

We focused on a group of downregulated genes, namely: SERPINA3, NNMT, LSM10, RCAN, SMARCA2, PSPH, $H 3 F 3 B, G L D C$, and TPMT, because their products have been linked to tumorigenesis. The expression of those genes was validated by qPCR in independent samples and compared with those in nontransformed melanocytes. The microarray findings were confirmed in case of six out of nine tested genes in both cell lines. In most cases, STAT3 knockdown partially reduced the expression of these genes to the levels detected in melanocytes (Fig. 3c).

\section{Detection of putative STAT3-binding sites in promoters of differentially expressed genes and validation of STAT3 occupancy by ChIP}

To identify STAT3-binding sites in the promoter regions of the differentially expressed genes, we searched for STAT-binding motifs that overlap with experimentally defined marks of active cis-regulatory regions e.g., transcription factor-binding sites, DNase1 hypersensitivity sites or activating epigenetic modifications (H3K27ac, H3K9me1) taken from Ensembl funcgen [32]. To confirm that STAT3 and active P-STAT3 bind to the putative STAT-binding sites in the promoter region of selected genes, we performed chromatin immunoprecipitation (ChIP) analysis. Figure 4a shows the promoters of the selected genes with marked positions of primers (orange arrows) used to determine STAT3 binding by ChIP-PCR. Primers were designed to flank the putative STAT-binding sites and generate $\sim 150 \mathrm{bp}$ of PCR fragments. Genespecific PCRs were performed on input samples, samples precipitated with antibody anti-pSTAT3 (Y705), anti-total 


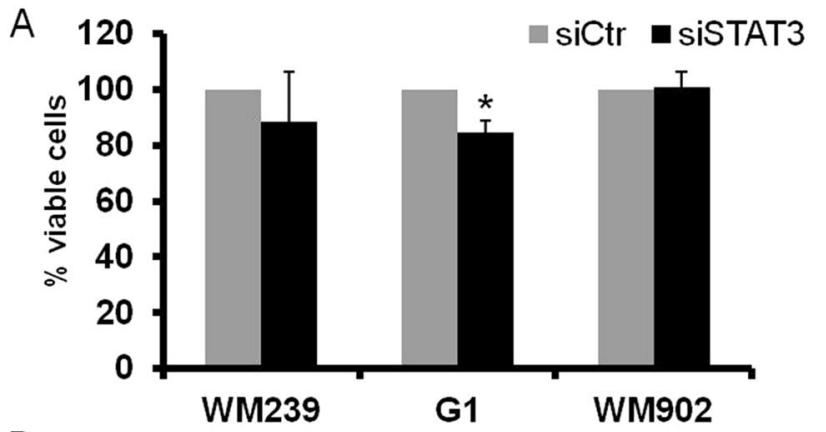

B
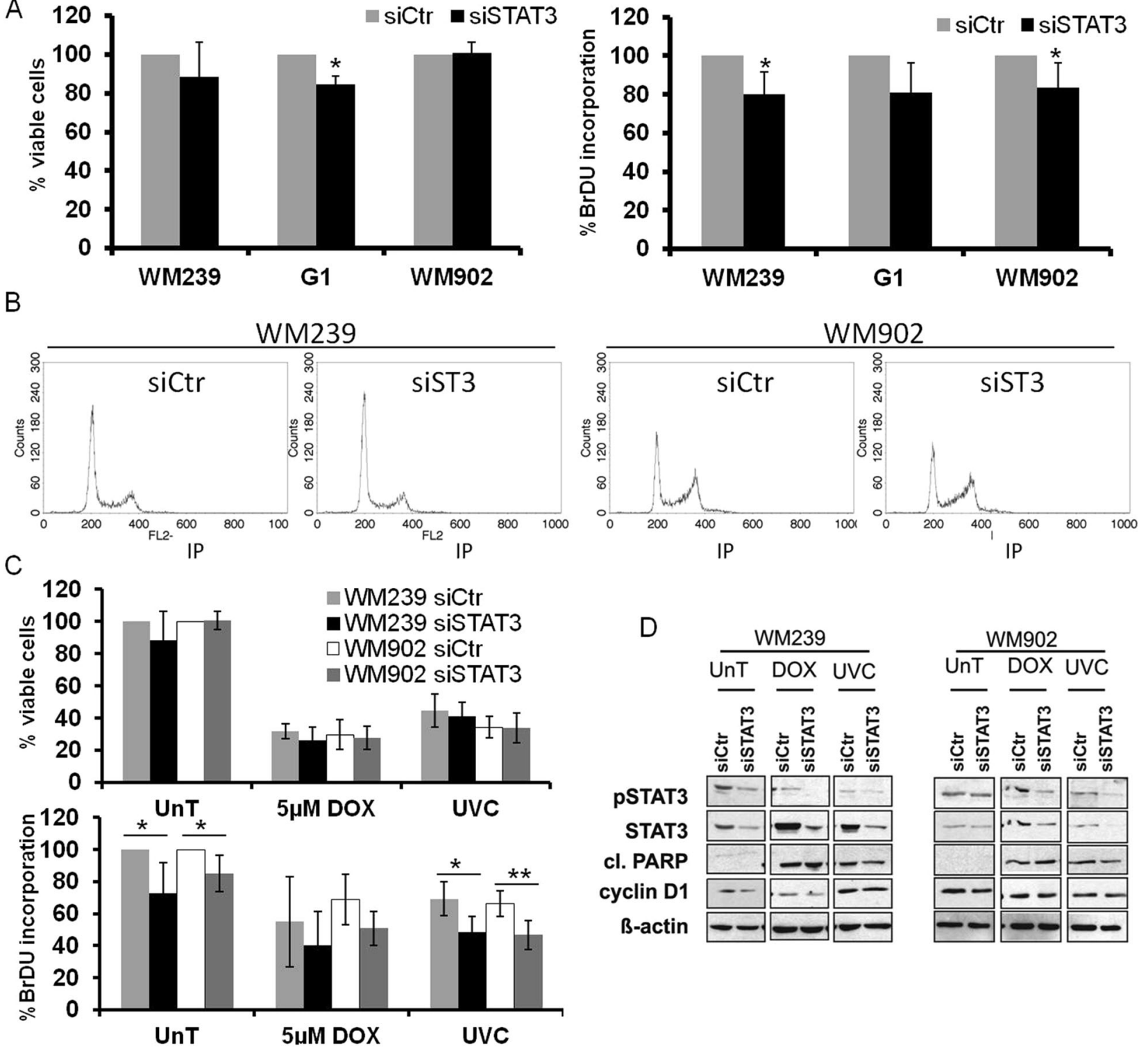

Fig. 2 The effect of STAT3 knockdown on viability, proliferation, and treatment responses of melanoma cells. a MTT metabolism test and BrdU incorporation test were used to evaluate viability and proliferation of melanoma cells, respectively, $72 \mathrm{~h}$ after the transfection with siSTAT3 or control siRNA (taken as $100 \%$ ). The data represent mean \pm s.d. from four independent experiments; statistical significance calculated by Student's $t$ test, $p<0.05$ were considered significant. b Representative histograms show distribution and percentages of cells in various phases of the cell cycle determined by flow cytometry. The cells were transfected with siRNAs, and $72 \mathrm{~h}$ later cells were collected, stained with propidium iodide, and analyzed by flow cytometry. The percentage of cells in the sub-G1, G1, S, and G2/M populations was determined by CellQuest software. c Evaluation of doxorubicin or UVC effects on STAT3-depleted cells. Percentages of living melanoma cells transfected with siSTAT3 or control siRNA and treated 48

STAT3 antibody, and with a control antibody (IgG). Binding of phosphorylated STAT3 to all six selected genes in WM239 and G1 melanoma cells was confirmed. This was additionally corroborated by detecting total h later with $5 \mu \mathrm{M}$ doxorubicin or irradiated with UVC light are presented. MTT metabolism test was performed $24 \mathrm{~h}$ after treatment. The results are related to values obtained for the cells transfected with siCtr. The data represent means \pm s.d. from four independent experiments; statistical analysis was done by Student's $t$ test, $p<0.05$ are considered significant. Lower panel shows the analysis of cell proliferation in the same cultures. BrDU incorporation assay was performed $24 \mathrm{~h}$ after treatment. d Immunoblots show the levels of STAT3, the cell cycle-regulatory protein-CYCLIN D1, and the levels of cleaved PARP in control and STAT3-depleted cells after treatments in two melanoma cell lines. Total protein extracts were collected $24 \mathrm{~h}$ post treatment, whole-cell lysates $(30 \mu \mathrm{g})$ were subjected to SDSPAGE, blotted, and probed with various antibodies; detection of $\beta$ actin was used as a protein loading control

STAT3 binding to selected genes using antibody recognizing STAT3. The ChIP-PCR results validated the selected genes as direct STAT3 targets in melanoma cells (Fig. 4b). 
A

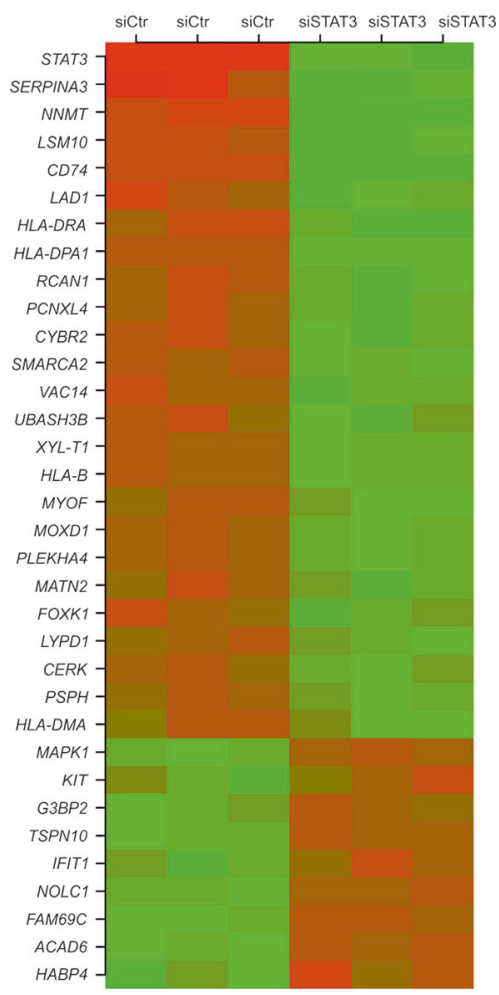

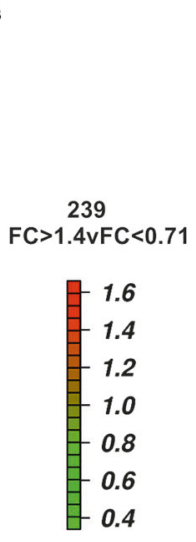

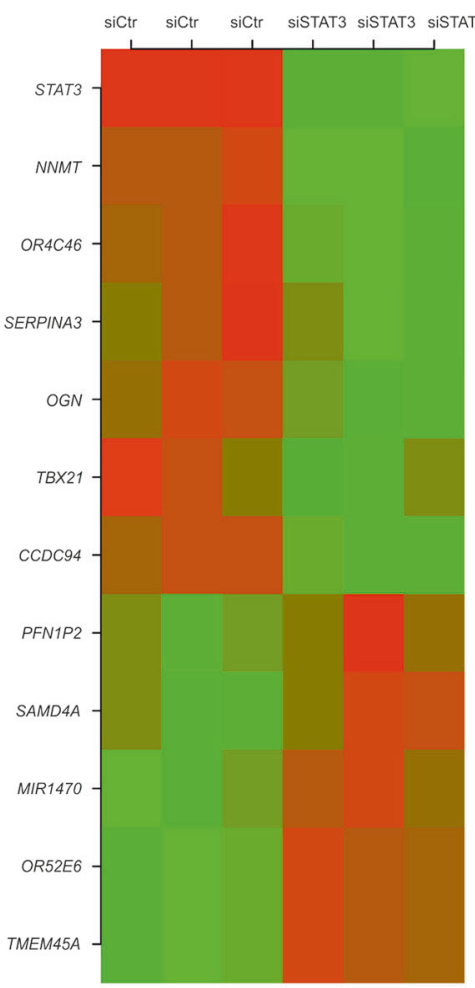

G1

FC $>1.4 v F C<0.71$

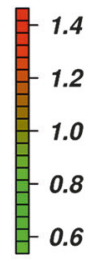

B
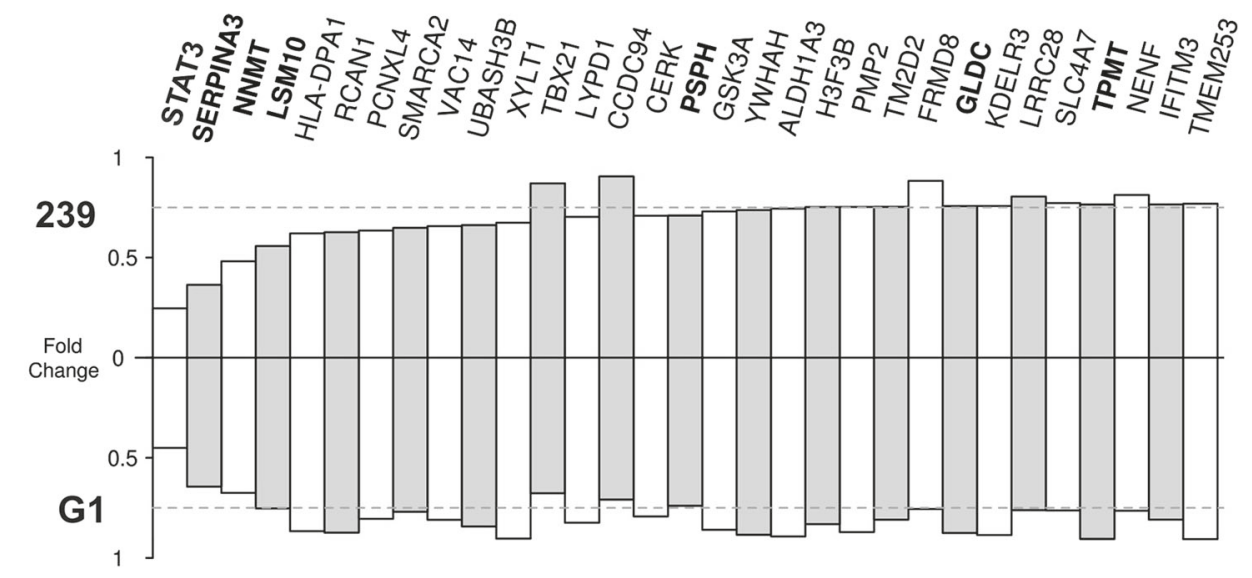

C
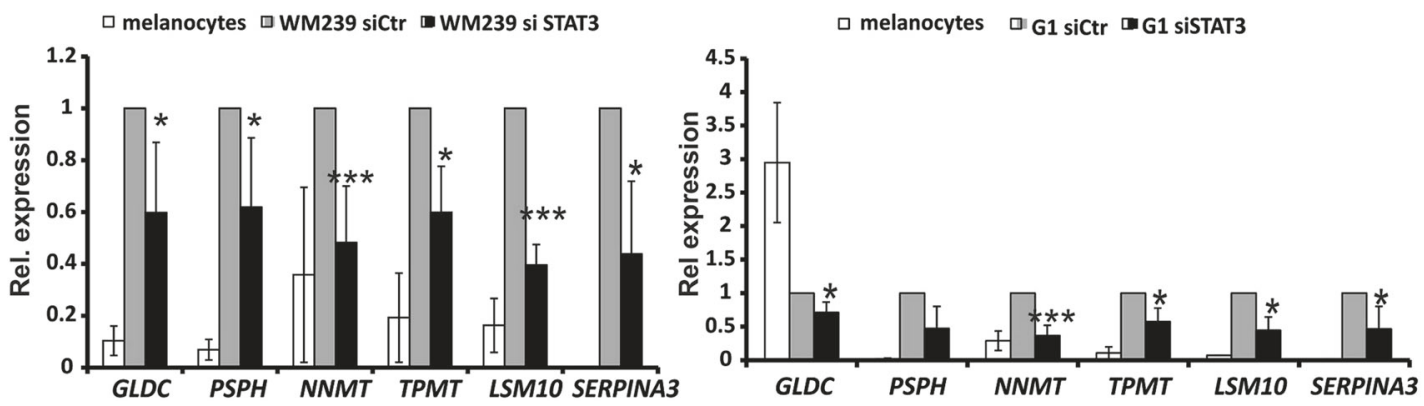
Fig. 3 Identification of STAT3-dependent genes by global expression profiling. a Global changes in gene expression induced by STAT3 knockdown in WM239 (left panel) and G1 (right panel) melanoma cell lines transfected with $\operatorname{siCtr}(n=3)$ and siSTAT3 $(n=3)$. Heatmaps represent expression levels of differentially expressed genes (fold change $>1.4$ or $<0.71$ ) between siCtr- and siSTAT3-treated melanoma cells. The levels of gene expression are indicated on a color scale, with red corresponding to the highest level of expression and green corresponding to the lowest level. b Barplot shows expression of genes at least $10 \%$ downregulated in both treated cell lines or 30\% downregulated in at least one cell line, genes selected for further analysis are marked in bold. c Validation of selected genes in melanocytes, siCtr, or siSTAT3-treated melanoma cells. RNA was isolated from independent cultures, and gene expression was determined with qPCR, $n=3$. Statistical significance was calculated with Student's $t$ test, ${ }^{*} p<$ $0.05, * * * p<0.001$ are considered significant

\section{STAT3 and SERPINA3 regulate migration and invasion of melanoma cells}

We found that SERPINA3 is a novel transcriptional target of STAT3 in melanoma cells. SERPINA3 is a member of the serpin super-family of protease inhibitors also known as $\alpha 1$ antichymotrypsin $(\alpha 1-\mathrm{ACT})$ in humans and serpina 3 in mice. SERPINA3 overexpression has been observed in prostate, breast, and colon cancers in connection with regulation of tumor proliferation and progression [33, 34]. Therefore, we pursued studies on involvement of STAT3 and SERPINA3 in regulation of cancer cell migration and invasion. To determine if STAT3 knockdown affects cell migration, we used a scratch assay. Melanoma WM239 and G1 cells transfected with control or STAT3 siRNAs were plated and $24 \mathrm{~h}$ after transfection subjected to a scratch. Cells were fixed with $2 \%$ PFA $18 \mathrm{~h}$ after a scratch and stained with DAPI to quantify a number of cells migrating to a cell-free area. We demonstrate reduced migration of WM239 and G1 cells depleted of STAT3 when compared with controls (Fig. 5a). Quantification of the results shows significantly reduced migration of STAT3-depleted melanoma cells (Fig. 5b).

We used a quantitative Matrigel-invasion assay, in which the nuclei of WM239 and G1 melanoma migrating cells are stained with DAPI, and fluorescence is measured using laser scanning cytometry (LSC). Representative images show scanned Matrigel inserts with nuclei of invading cells (Fig. 6a). Invasion of mock and siCtr-transfected cells did not differ. Invasion of STAT3-depleted cells was significantly reduced when compared with controls, taken as $100 \%$ (Fig. $6 b)$.

To assess if SERPINA3 mediates STAT3 effects on invasion of WM239 and G1 melanoma cells, we determined invasion of cells that were transfected with SERPINA3 siRNA or with SERPINA3 overexpressed in melanoma cells depleted of STAT3. The number of cells invading the Matrigel increased significantly (almost threefold) in WM239 and G1 cells overexpressing SERPINA3 compared with controls. Invasion of STAT3depleted WM239 and G1 cells was significantly increased when SERPINA3 was overexpressed in these cells. The cells transfected with siRNA SERPINA3 showed a significant reduction in the number of invading cells (Fig. 7a). The upper part shows representative images of nuclei in transfected WM239 and G1 cell cultures; Fig. 7b shows quantification of the results from three experiments. The efficacy of STAT3 knockdown has been validated by western blotting (Fig. 7c). Notably, SERPINA3 expression was significantly reduced after STAT3 knockdown in five human melanoma patient-derived cell lines. The results demonstrate that overexpression of SERPINA3 enhances invasion and compensates for invasion impairment in STAT3-depleted cells. SERPINA3 knockdown reduced invasion of melanoma cells.

Activated STAT3 can promote cell migration and invasion of some cancer cells through enhancement of the MMP-2, MMP-9, MMP-14 gene expression, which may induce extracellular matrix remodeling and mesenchymal properties during cancer metastasis. The effect of STAT3 knockdown on the expression of $M M P-2, M M P-9$, and MMP-14 mRNAs was determined in WM239 and G1 cells. The expression of MMPs did not change in STAT3depleted cells (the supplementary Fig. S1). The results demonstrate that the inhibitory effect of STAT3 knockdown on melanoma invasion was likely mediated by STAT3dependent upregulation of SERPINA3 expression.

\section{Discussion}

Here we report identification of novel STAT3-dependent genes in melanoma cells and contribution of newly discovered STAT3-SERPINA3 axis to melanoma invasion. We also confirmed upregulation of STAT3 expression in metastatic melanoma cell lines and re-evaluated the role of STAT3 in regulation of melanoma cell viability and proliferation to explain some discrepancies in the existing data. We found that efficient knockdown of STAT3 does not change significantly the viability of STAT3-depleted human melanoma cells and only moderately reduces proliferation (Fig. 2a). The results were similar in three patient-derived melanoma cell cultures and were further corroborated by cell cycle analyses using flow cytometry.

In contrast to some reports, STAT3 knockdown in melanoma cells only moderately enhanced susceptibility to doxorubicin $(\sim 15 \%)$ and UVC radiation $(\sim 20 \%)$. Additional analysis using western blotting did not show significant differences in responses to antitumor treatments between controls and STAT3-depleted cells. All these results suggest a minor role of STAT3 in regulation of proliferation and cell 
A

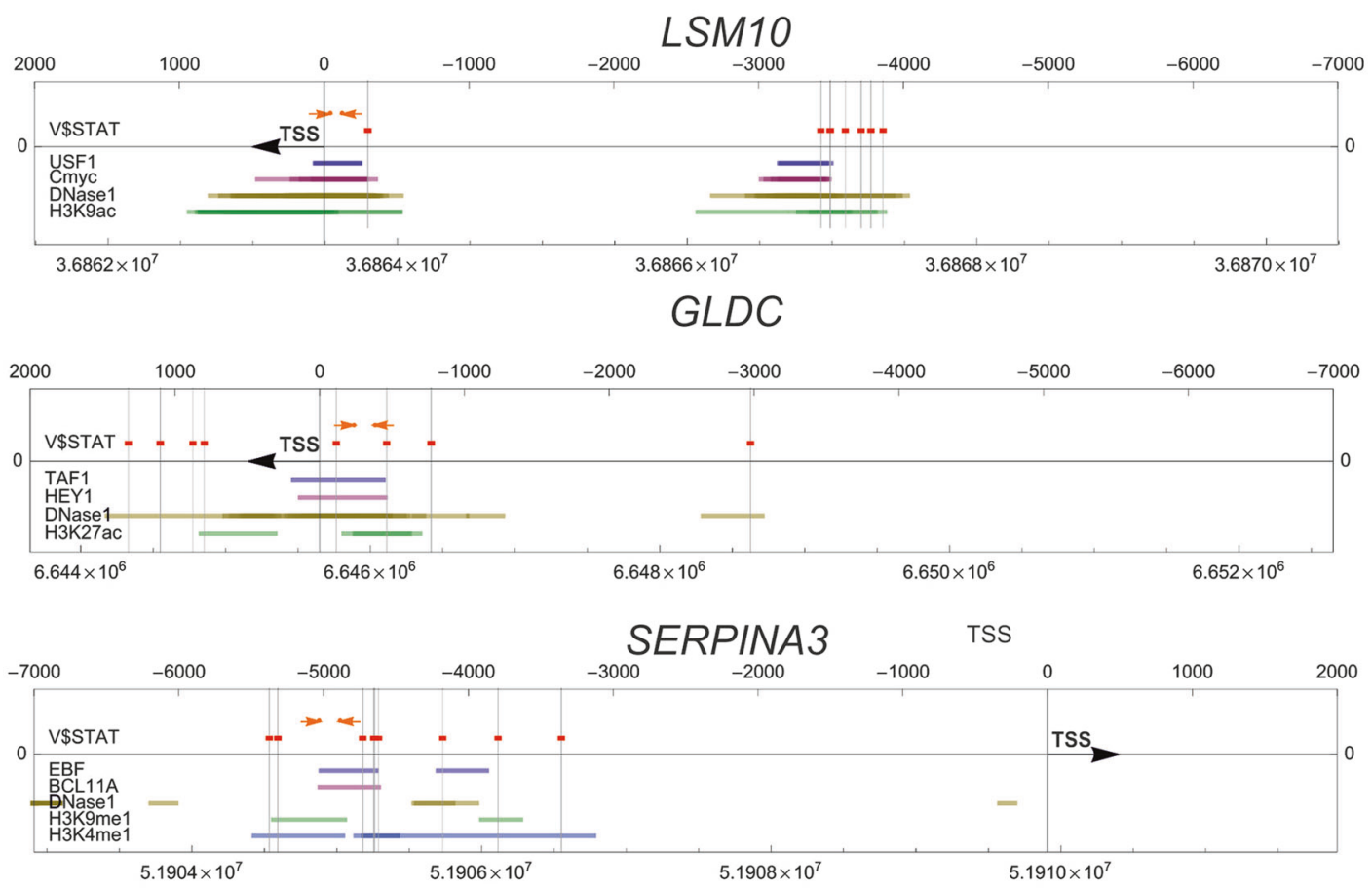

B

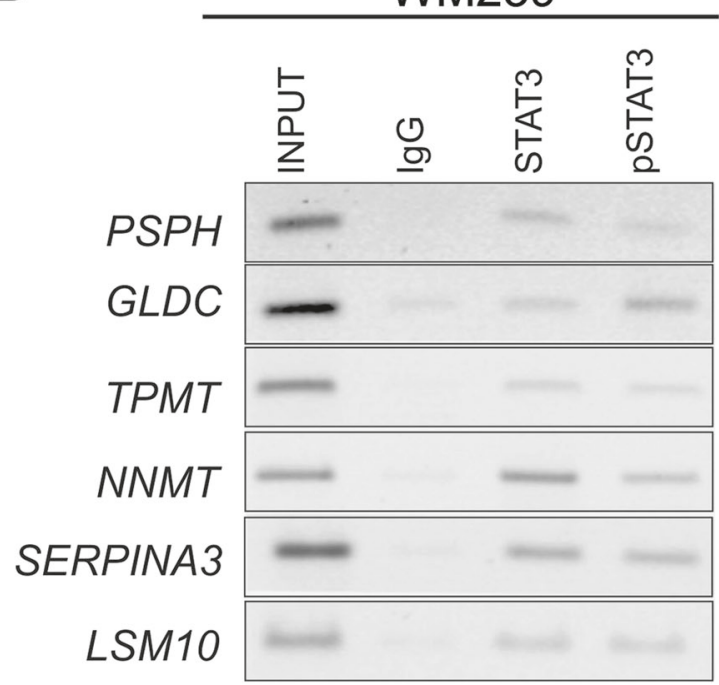

Fig. 4 Validation of STAT3 binding to the promoters of selected genes. a Computational analysis of promoter regions of selected genes revealed the presence of STAT motifs (in red) near the transcription start site (TSS). Yellow arrows mark positions of primers design to validate STAT3 binding. b ChIP assay was performed with untreated

viability of human melanoma cells. Our results are in disagreement with some studies showing a role of STAT3 in viability of human melanoma cells, but in the previous studies nonspecific tools such as inhibitors of Jak/STAT or Akt signaling were used to block STAT3 function. A recent study using structurally modified indirubin derivatives as inhibitors of STAT3 phosphorylation showed induction of
G1

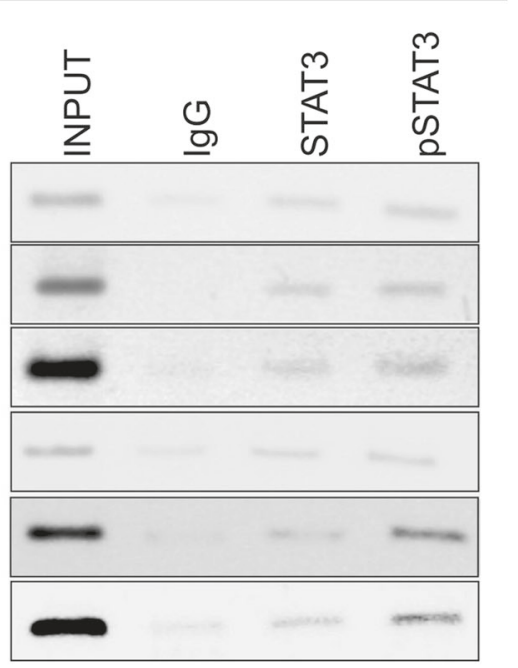

WM239 and G1 cells with antibody against pSTAT3, STAT3 total, and control IgG. Non immunoprecipitated chromatin was used as total input control. PCR primers were designed to include putative STAT3binding sites in the promoter of selected genes. The data are representative for three independent experiments

apoptosis in two human melanoma cell lines and the lack of effects in others [35]. The lack of STAT3 could be compensated by the other survival pathways, such as NFkB signaling, as we previously demonstrated in lung cancer cells [20].

Initially, we used pre-designed gene expression profilers Cyclins, Cell Cycle Regulation, and JAK-STAT 
Fig. 5 STAT3 knockdown affects melanoma cell migration. a Cell migration was determined with a scratch assay. After transfection with control or STAT3-specific siRNAs, confluent monolayers of WM239 and G1 cells were mechanically disrupted with a scratch using a sterile $100 \mu \mathrm{l}$ tip, then photographed immediately $(0 \mathrm{~h})$ and at $18 \mathrm{~h}$ after scratching. The assay was done in three separate experiments, and two experiments are presented. b Cells were incubated for $18 \mathrm{~h}$ after the scratch, fixed with $2 \%$ paraformaldehyde, stained with DAPI and analyzed by fluorescence microscopy (10 photos were taken per condition). The photos were then quantified using Image J software. The bar chart shows numbers of cells that had migrated among cells treated with siSTAT3 compared with the control. The results shown means \pm s.d. from three experiments, in duplicate. Statistical analysis was done by Student's $t$ test, ${ }^{*} p<0.05$, $* * * p<0.001$ are considered significant
A

WM239
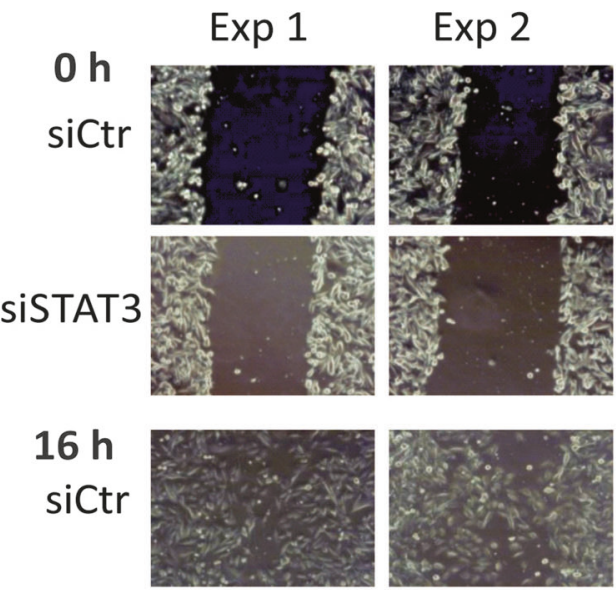

SISTAT3
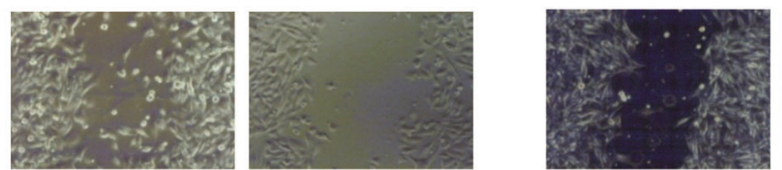

G1
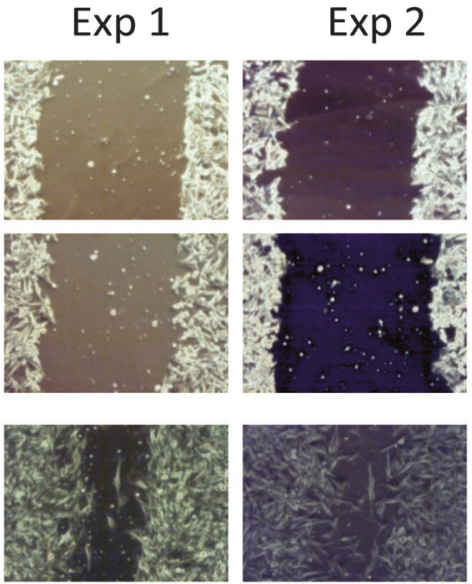

B

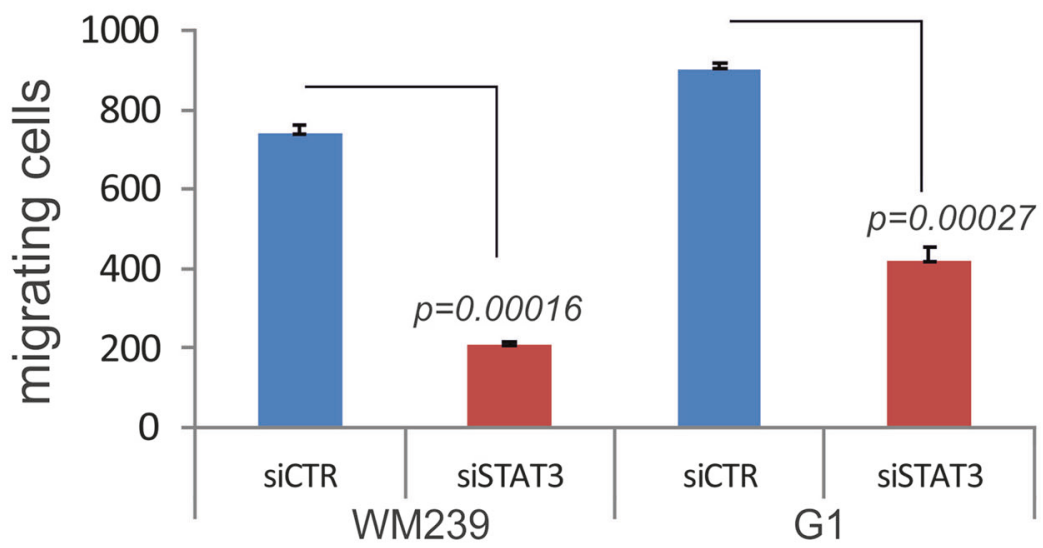

containing many known STAT-regulated genes, but out of 130 tested genes only a small set of genes was significantly altered in STAT3-depleted cells; mostly in G1 malignant melanoma cells. Those genes were functionally connected with cell cycle regulation and could mediate reduction of cell proliferation observed in STAT3depleted cells (Figs. 1, 2). The expression of those genes was upregulated in melanoma cells in comparison with melanocytes, and STAT3 knockdown partly reduced their expression to the levels observed in melanocytes. A weak functional response after STAT3 knockdown may be due to a dual role of STAT3. Knockdown of STAT3 not only decreased expression of pro-survival genes, such as $E G F, V E G F$, and $C C N A 1$, but also reduced expression of suppressor genes, namely: $C D K N 2 A, C D K N 2 B, E 2 F 4$, and STAT1. A list of altered genes for a given cell line is illustrated by heatmap in the Fig. 1d. Altogether, the presented results suggest that STAT3 plays a pro- oncogenic role in melanoma cells, but is not essential for basal survival.

To determine STAT3-dependent transcriptional network, we studied global gene expression patterns in control (siCtr) and STAT3-depleted cells in two metastatic melanoma cell lines. The Fig. 3 presents genes that were significantly differentially expressed after STAT3 knockdown: more than 30 genes in WM239 cells and 11 in G1 cells. A set of transcriptionally altered genes and their fold changes were rather small. These results are in contrast to the studies presenting STAT3 as a major oncogenic transcription factor in cancer [9-15]. Moreover, the computational analysis of STAT3-dependent genes with the use of the consistent signal transduction algorithm [36] did not reveal any altered pathway upon STAT3 knockdown. This could be due to already mentioned compensation by other STAT family members or compensatory NFkB activation, as we previously demonstrated [37]. Nevertheless, we identified a set 


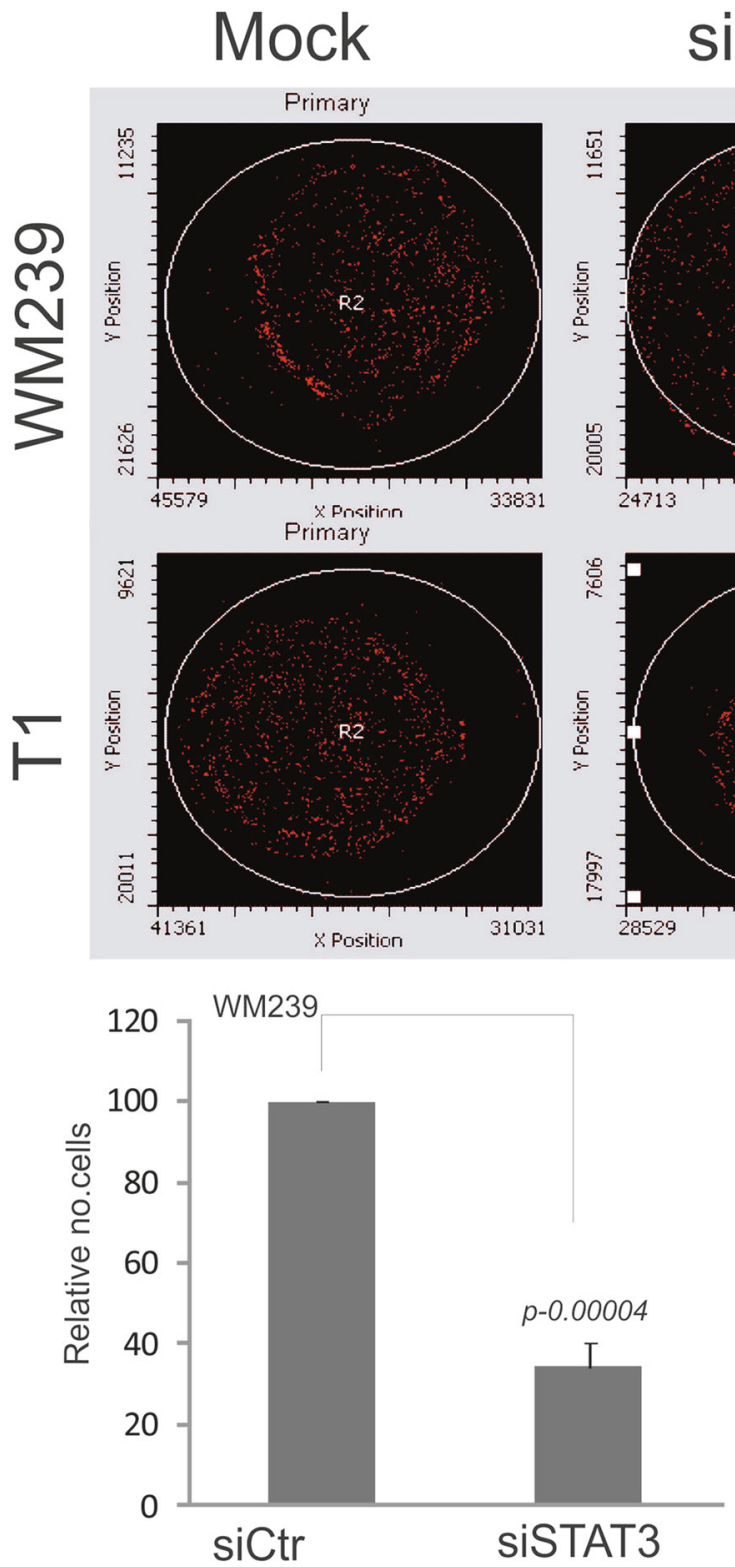

Fig. 6 STAT3 knockdown impairs invasion of WM239 and G1 melanoma cells. a Invasion of melanoma cells was evaluated by in Matrigel assay coupled with laser scanning cytometry (LSC). WM239 and G1 cells transfected with siCtr or siSTAT3 were plated on a Matrigel-coated polycarbonate membrane Transwell Inserts $48 \mathrm{~h}$ after transfection. After $18 \mathrm{~h}$, cells invading through the Matrigel were fixed

of genes with expression altered by STAT3-depleted cells. It includes known STAT3 target genes, such as those of belonging to the JAK-STAT pathway, JAK-STAT cascade involved in the growth hormone signaling pathway, and interleukin-6-mediated signaling pathway. We found genes related to positive regulation of the fibroblast proliferation or nerve growth factor receptor signaling pathway, which is consistent with results of gene profiler analysis where $E G F$, $E G F R$, VEGF, and SOCS1 expression was reduced in STAT3-depleted cells. Moreover, genes belonging to Gene

\section{SISTAT3}
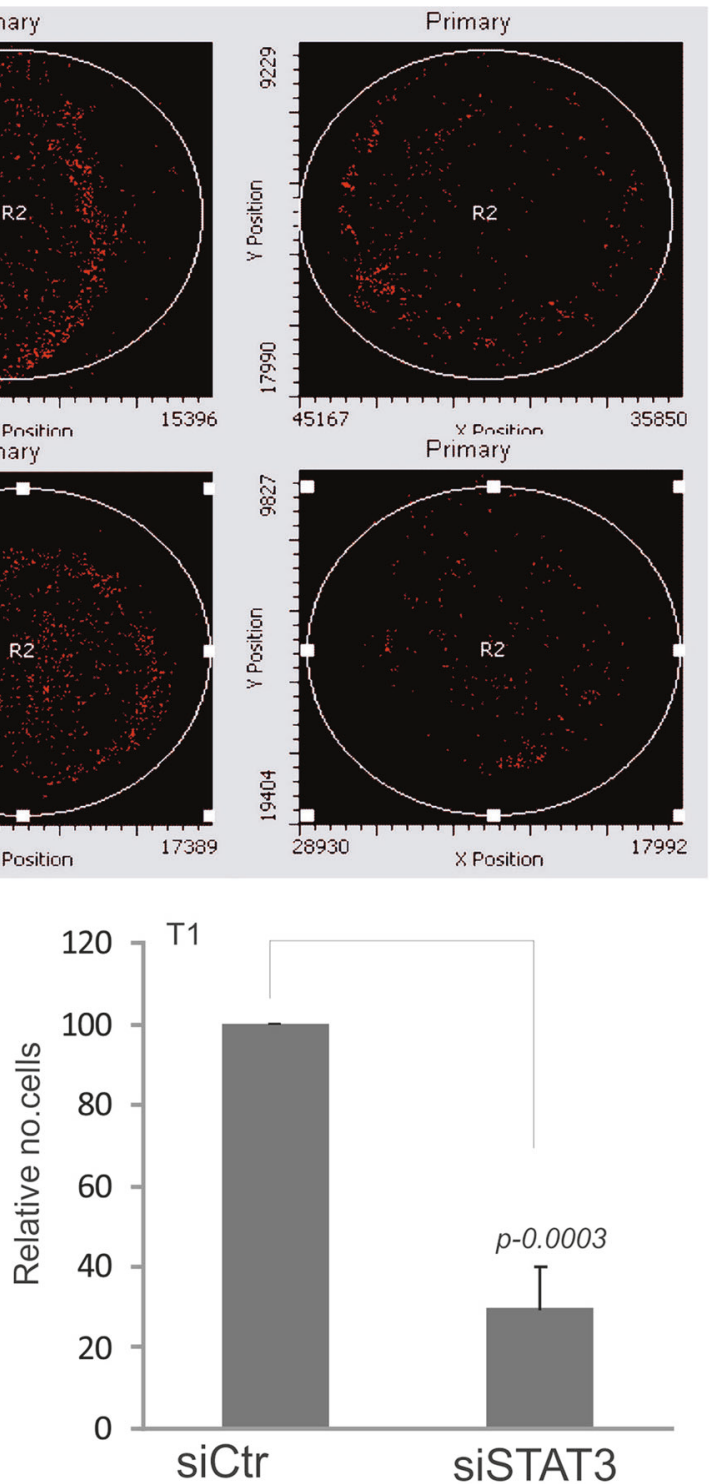

with 95\% methanol, their nuclei stained with DAPI, and quantified using LSC. b The histogram shows the significance difference in invasion of STAT3-depleted cells compared with the siCtr group. The results shown as means from three experiments done in duplicate. Statistical analysis was done by Student's $t$ test, $* p<0.05$, ***p $p<$ 0.001 are considered significant

Ontology terms such as "negative regulation of DNA damage response, signal transduction by p53 class mediator" were slightly altered upon STAT3 knockdown.

To find STAT3-dependent genes in metastatic melanoma cells, we selected 30 genes consistently, differentially expressed in two STAT3-depleted cell lines (Fig. 5b). From this panel of genes, we selected those potentially involved in tumorigenesis: SERPINA3, NNMT, TPMT, LSM10, H3F3B, RCAN1, SMARCA2, GLDC, and PSPH. Real-time qPCR analysis on independent cultures confirmed the 


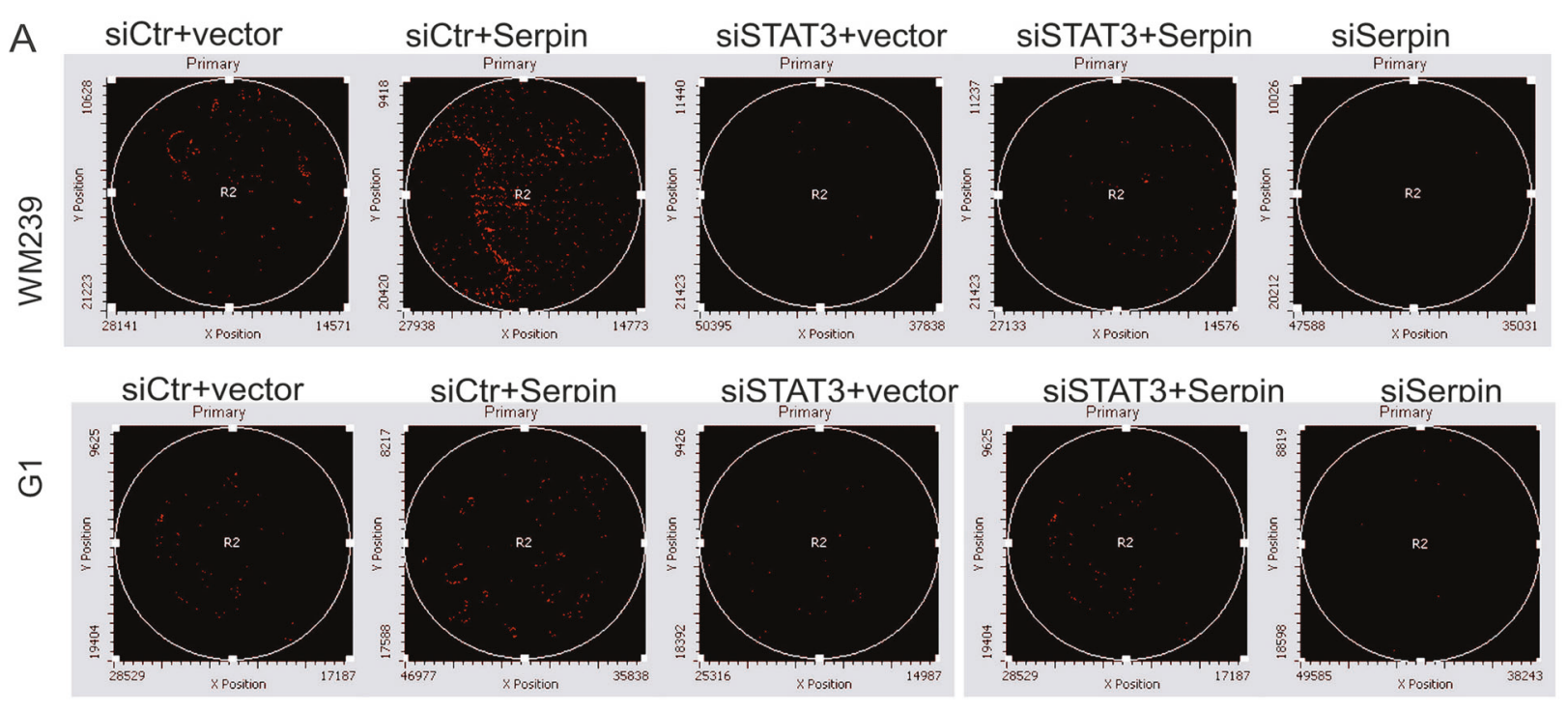

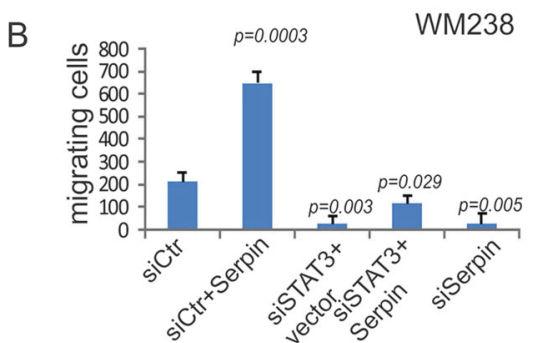

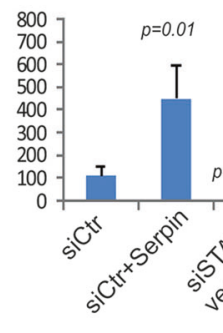

Fig. 7 The effects of manipulation of STAT3 and/or SERPINA3 levels on melanoma cell invasion. a Representative images of Matrigelinvading melanoma cells under various conditions. WM239 and G1 cells were transfected with control, STAT3, or SERPINA3-specific siRNAs; with or without overexpression of the SERPINA3 plasmid. Cells were platted on a Matrigel-coated inserts, $48 \mathrm{~h}$ after transfection. After additional $18 \mathrm{~h}$, cells invading through the Matrigel were then fixed with 95\% methanol, and their nuclei were stained with DAPI and quantified using laser scanning cytometry. b Quantification of the effects of manipulation of STAT3 and SERPINA3 levels on melanoma cell invasion. The histograms indicate the number of cells that had

expression changes for six out of nine genes. STAT3 knockdown partially reverted the expression of tested genes in melanoma cells to their levels detected in melanocytes (Fig. 3c). By using chromatin immunoprecipitation, we verified direct STAT3 binding to regulatory elements in the promoters of all selected genes (Fig. 4b).

Those genes are for the first time identified as STAT3 direct targets in melanoma cells, only NNMT was earlier presented as directly regulated by STAT3 in Hep-G2 cells and SW480 colon cancer cells upon stimulation with IL-6 [38]. A recent study shows that nicotinamide $\mathrm{N}$ methyltransferase (NNMT), an enzyme that catalyzes the $\mathrm{N}$-methylation of nicotinamide and pyridine compounds and participates in drug metabolism, is overexpressed in malignant melanoma [39], which is in agreement with the presented data. All of the examined genes are functionally involved in tumorigenesis and tumor progression [40-43]. invaded the Matrigel. The histogram presents the significant differences in invasion of cells with manipulated STAT3 and SERPINA3 levels compared with the control group ( $n=3$ experiments, in duplicate). c Representative immunoblots show the levels of STAT3, phSTAT3, and SERPINA3 in five melanoma cell lines transfected with control or STAT3 specific siRNAs; all cell lines were derived from melanoma patients. Notable are the reduced levels of SERPINA3 in all tested cell lines after successful knockdown of STAT3. Detection of $\beta$ ACTIN on the same immunoblots ensured equal loading of total protein extracts

We focused on SERPINA3, which encodes alpha-1 antichymotrypsin, a serine protease inhibitor. High expression of SERPINA3 correlates with stage of cancer and poor prognosis in colon cancer and melanoma [44, 45]. SERPINA3 expression is elevated in patients with metastatic melanoma, predicts poor survival and its role in promoting malignant properties of melanoma cells has been postulated [45]. We found that STAT3 binds to the SERPINA3 gene promoter and regulates its expression. Notably, SERPINA3 expression was significantly reduced after STAT3 knockdown in five human melanoma patient-derived cell lines (Fig. 7b). It shows that dependence of SERPINA3 expression on STAT3 is a common phenomenon in melanomas. We studied migration and invasion of melanoma cells after STAT3 knockdown and found that knockdown of STAT3 strongly impaired migration and invasion of melanoma cells. Also knockdown of SERPINA3 had the 
inhibitory effect on invasion of melanoma cells, while overexpression of SERPINA3 increased invasion and restored invasion of STAT3-depleted melanoma cells. As overexpression of SERPINA3 in STAT3-depleted cells restored the invasion (Fig. 7a, b), we conclude that STAT3dependent induction of SERPINA3 contributes to the enhanced invasion of metastatic melanoma cells. The role of active STAT3 in invasion is best presented when T1 and G1 melanoma cell lines are compared. Those cells were derived from the primary lesion (T1) and from an invaded lymph node (G1) of the same patient [46] and the active STAT3 level is high in metastatic G1 melanoma cells. Our results are corroborated by a recent study showing the high expression of SERPINA3 in melanomas, and its association with the worse survival in melanoma patients. It has been proposed that SERPINA3 expression could be a prognostic factor to predict melanoma patient clinical outcome [47].

In conclusion, we identified a set of new STAT3dependent genes in metastatic melanoma cells, some of which regulate melanoma cell migration and invasion. Our results demonstrate new functions of STAT3 in regulating tumor invasion. Although, STAT3 regulates genes implicated in the regulation of cell cycle and cell survival, its knockdown has the minor effect on those processes likely due to the presence of compensatory pathways. The STAT3-dependent upregulation of SERPINA3 expression is a novel mechanism of regulation of melanoma invasion.

Acknowledgements This work was supported by M345/N-INCA/ 2008 from the Polish Ministry of Science and Higher Education (BK), Foundation for Polish Science International PhD Program (KR) and National Science Center grant 2011/03//N/NZ3/05897 (DWK).

\section{Compliance with ethical standards}

Conflict of interest The authors declare that they have no conflict of interest.

Publisher's note: Springer Nature remains neutral with regard to jurisdictional claims in published maps and institutional affiliations.

\section{References}

1. Wang T, Niu G, Kortylewski M, Burdelya L, Shain K, Zhang S, et al. Regulation of the innate and adaptive immune responses by Stat-3 signaling in tumor cells. Nat Med. 2004;10:48-54.

2. Yu H, Kortylewski M, Pardoll D. Crosstalk between cancer and immune cells: role of STAT3 in the tumour microenvironment. Nat Rev Immunol. 2007;7:41-51.

3. Catlett-Falcone R, Landowski TH, Oshiro MM, Turkson J, Levitzki A, Savino R, et al. Constitutive activation of Stat3 signaling confers resistance to apoptosis in human U266 myeloma cells. Immunity. 1999;10:105-15.

4. Bromberg J. Stat proteins and oncogenesis. J Clin Invest. 2002;109:1139-42.

5. Masuda M, Suzui M, Yasumatu R, Nakashima T, Kuratomi Y, Azuma K, et al. Constitutive activation of signal transducers and activators of transcription 3 correlates with cyclin D1 overexpression and may provide a novel prognostic marker in head and neck squamous cell carcinoma. Cancer Res. 2002;62:3351-5.

6. Karni R, Jove R, Levitzki A. Inhibition of pp60c-Src reduces Bcl$\mathrm{XL}$ expression and reverses the transformed phenotype of cells overexpressing EGF and HER-2 receptors. Oncogene. 1999;18:4654-62.

7. Mahboubi K, Li F, Plescia J, Kirkiles-Smith NC, Mesri M, Du Y, et al. Interleukin-11 up-regulates survivin expression in endothelial cells through a signal transducer and activator of transcription3 pathway. Lab Invest. 2001;81:327-34.

8. Zhao M, Gao F-H, Wang J-Y, Liu F, Yuan HH, Zhang WY, et al. JAK2/STAT3 signaling pathway activation mediates tumor angiogenesis by upregulation of VEGF and bFGF in non-smallcell lung cancer. Lung Cancer. 2011;73:366-74.

9. Buettner R, Mora LB, Jove R. Activated STAT signaling in human tumors provides novel molecular targets for therapeutic intervention. Clin Cancer Res. 2002;8:945-54.

10. Haura EB, Turkson J, Jove R. Mechanisms of disease: insights into the emerging role of signal transducers and activators of transcription in cancer. Nat Clin Pract Oncol. 2005;2:315-24.

11. Kortylewski M, Jove R, Yu H. Targeting STAT3 affects melanoma on multiple fronts. Cancer Metastasis Rev. 2005;24:315-27.

12. Gao L, Zhang L, Hu J, Shao Y, Zhao D, Kalvakolanu DV, et al. Down-regulation of signal transducer and activator of transcription 3 expression using vector-based small interfering RNAs suppresses growth of human prostate tumor in vivo. Clin Cancer Res. 2005;11:6333-41.

13. Huang C, Yang G, Jiang T, Cao J, Huang KJ, Qiu ZJ. Downregulation of STAT3 expression by vector-based small interfering RNA inhibits pancreatic cancer growth. World J Gastroenterol. 2011;17:2992-3001.

14. Klosek SK, Nakashiro K, Hara S, Goda H, Hamakawa H. Stat3 as a molecular target in RNA interference-based treatment of oral squamous cell carcinoma. Oncol Rep. 2008;20:873-8.

15. Leong PL, Andrews GA, Johnson DE, Dyer KF, Xi S, Mai JC, et al. Targeted inhibition of Stat 3 with a decoy oligonucleotide abrogates head and neck cancer cell growth. Proc Natl Acad Sci USA. 2003;100:4138-43.

16. Li J, Piao Y-F, Jiang Z, Chen L, Sun HB. Silencing of signal transducer and activator of transcription 3 expression by RNA interference suppresses growth of human hepatocellular carcinoma in tumor-bearing nude mice. World J Gastroenterol. 2009;15:2602-8.

17. Ni Z, Lou W, Leman ES, Gao AC. Inhibition of constitutively activated Stat 3 signaling pathway suppresses growth of prostate cancer cells. Cancer Res. 2000;60:1225-8.

18. Zhang X, Zhang J, Wang L, Wei H, Tian Z. Therapeutic effects of STAT3 decoy oligodeoxynucleotide on human lung cancer in xenograft mice. BMC Cancer. 2007;7:149.

19. Adach-Kilon A, Swiatek-Machado K, Kaminska B, Dabrowski M. Signal transducer and activator of transcription 1 (Stat1) maintains basal mRNA expression of pro-survival stat3-target genes in glioma C6 cells. J Cell Biochem. 2011;112:3685-94.

20. Kulesza DW, Carré T, Chouaib S, Kaminska B. Silencing of the transcription factor STAT3 sensitizes lung cancer cells to DNA damaging drugs, but not to TNF $\alpha$ - and NK cytotoxicity. Exp Cell Res. 2013;319:506-16.

21. Zammarchi F, de Stanchina E, Bournazou E, Supakorndej T, Martires K, Riedel E, et al. Antitumorigenic potential of STAT3 alternative splicing modulation. Proc Natl Acad Sci USA. 2011;108:17779-84.

22. de la Iglesia N, Konopka G, Puram SV, Chan JA, Bachoo RM, You MJ et al. Identification of a PTEN-regulated STAT3 brain tumor suppressor pathway. Genes Dev. 2008;22:449-62. 
23. Dummer R, Hauschild A, Lindenblatt N, Pentheroudakis G, Keilholz U; ESMO Guidelines Committee. Melanoma: ESMO clinical practice guidelines for diagnosis, treatment and follow-up. Ann Oncol. 2015;26:126-32.

24. Niu G, Bowman T, Huang M, Shivers S, Reintgen D, Daud A, et al. Roles of activated Src and Stat3 signaling in melanoma tumor cell growth. Oncogene. 2002;21:7001-10.

25. Wu ZS, Cheng XW, Wang XN, Song NJ. Prognostic significance of phosphorylated signal transducer and activator of transcription 3 and suppressor of cytokine signaling 3 expression in human cutaneous melanoma. Melanoma Res. 2011;21:483-90.

26. Xie T-X, Wei D, Liu M, Gao AC, Ali-Osman F, Sawaya R, et al. Stat3 activation regulates the expression of matrix metalloproteinase- 2 and tumor invasion and metastasis. Oncogene. 2004;23:3550-60.

27. Xie T, Huang F-J, Aldape KD, Kang SH, Liu M, Gershenwald JE, et al. Activation of stat 3 in human melanoma promotes brain metastasis. Cancer Res. 2006;66:3188-96.

28. Lee I, Fox PS, Ferguson SD, Bassett R, Kong LY, Schacherer $\mathrm{CW}$, et al. The expression of p-STAT3 in stage IV melanoma: risk of CNS metastasis and survival. Oncotarget. 2012;3:336-44.

29. Kang K, Robinson GW, Hennighausen L. Comprehensive metaanalysis of signal transducers and activators of transcription (STAT) genomic binding patterns discerns cell-specific cis-regulatory modules. BMC Genomics. 2013;14:4.

30. Cao HH, Chu JH, Kwan HY, Su T, Yu H, Cheng CY, et al. Inhibition of the STAT3 signaling pathway contributes to apigenin-mediated anti-metastatic effect in melanoma. Sci Rep. 2016;25:21731.

31. Dunning MJ, Smith ML, Ritchie ME, Tavaré S. beadarray: R classes and methods for Illumina bead-based data. Bioinformatics. 2007;23:2183-4.

32. Krystkowiak I, Lenart J, Debski K, Kuterba P, Petas M, Kaminska B, et al. Nencki Genomics Database-Ensembl funcgen enhanced with intersections, user data and genome-wide TFBS motifs. Database (Oxford). 2013;2013:bat069.

33. Higashiyama M, Doi O, Yokouchi H, Kodama K, Nakamori S, Tateishi R. Alpha-1-antichymotrypsin expression in lung adenocarcinoma and its possible association with tumor progression. Cancer. 1995;76:1368-76.

34. Baker C, Belbin O, Kalsheker N, Morgan K. SERPINA3 (aka alpha-1-antichymotrypsin). Front Biosci. 2007;12:2821-35.

35. Liu L, Nam S, Tian Y, Yang F, Wu J, Wang Y, et al. 6-Bromoindirubin-3'-oxime inhibits JAK/STAT3 signaling and induces apoptosis of human melanoma cells. Cancer Res. 2011;71:3972-9.
36. Mieczkowski J, Swiatek-Machado K, Kaminska B. Identification of pathway deregulation-gene expression based analysis of consistent signal transduction. PLoS ONE. 2012;7:e41541.

37. Ramji K, Kulesza DW, Chouaib S, Kaminska B. Off-target effects of plasmid-transcribed shRNAs on NFKB signaling pathway and cell survival of human melanoma cells. Mol Biol Rep. 2013;40:6977-86.

38. Tomida M, Ohtake H, Yokota T, Kobayashi Y, Kurosumi M. Stat3 up-regulates expression of nicotinamide $\mathrm{N}$-methyltransferase in human cancer cells. J Cancer Res Clin Oncol. 2008;134:551-9.

39. Ganzetti G, Sartini D, Campanati A, Rubini C, Molinelli E, Brisigotti $\mathrm{V}$, et al. Nicotinamide $\mathrm{N}$-methyltransferase: potential involvement in cutaneous malignant melanoma. Melanoma Res. 2018;28:82-8.

40. Zhang WC, Shyh-Chang N, Yang H, Rai A, Umashankar S, Ma S, et al. Glycine decarboxylase activity drives non-small cell lung cancer tumor-initiating cells and tumorigenesis. Cell. 2012;148:259-72.

41. Santamaria M, Pardo-Saganta A, Alvarez-Asiain L, Di Scala M, Qian C, Prieto J, et al. Nuclear $\alpha 1$-antichymotrypsin promotes chromatin condensation and inhibits proliferation of human hepatocellular carcinoma cells. Gastroenterology. 2013;144:818-28.

42. Grek CL, Townsend DM, Uys JD, Manevich Y, Coker WJ 3rd, Pazoles CJ, et al. S-glutathionylated serine proteinase inhibitors as plasma biomarkers in assessing response to redox-modulating drugs. Cancer Res. 2012;72:2383-93.

43. Tang S-W, Yang T-C, Lin W-C, Chang WH, Wang CC, Lai MK, et al. Nicotinamide $\mathrm{N}$-methyltransferase induces cellular invasion through activating matrix metalloproteinase- 2 expression in clear cell renal cell carcinoma cells. Carcinogenesis. 2011;32:138-45.

44. Dimberg J, Ström K, Löfgren S, Zar N, Hugander A, Matussek A. Expression of the serine protease inhibitor serpinA3 in human colorectal adenocarcinomas. Oncol Lett. 2011;2:413-8.

45. Wang Y, Jiang H, Dai D, Su M, Martinka M, Brasher P, et al. Alpha 1 antichymotrypsin is aberrantly expressed during melanoma progression and predicts poor survival for patients with metastatic melanoma. Pigment Cell Melanoma Res. 2010;23:575-8.

46. Gassara A, Messai Y, Gaudin C, Abouzahr S, Jalil A, DiarraMehrpour M, et al. The decreased susceptibility of metastatic melanoma cells to killing involves an alteration of CTL reactivity. Int J Oncol. 2006;29:155-61.

47. Zhou J, Cheng Y, Tang L, Martinka M, Kalia S. Up-regulation of SERPINA3 correlates with high mortality of melanoma patients and increased migration and invasion of cancer cells. Oncotarget. 2017;8:18712-25. 